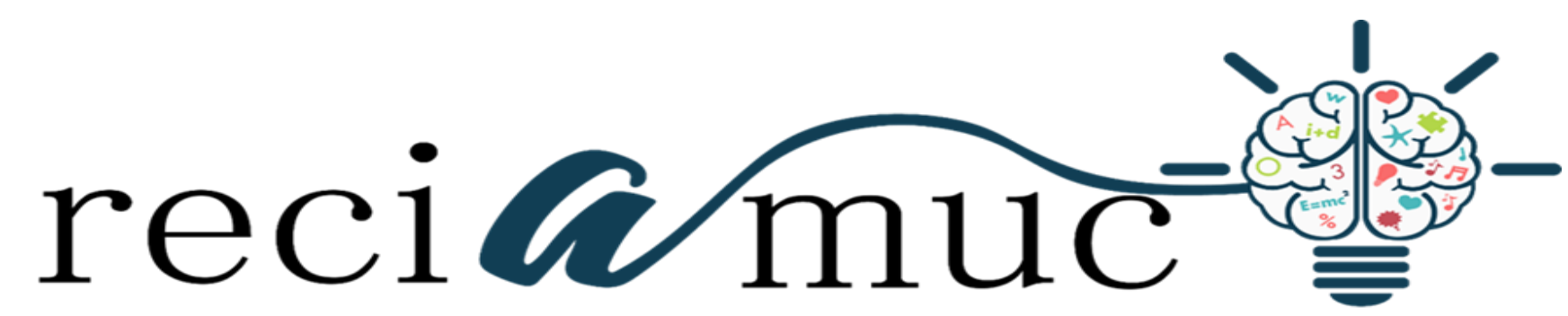

Revista científica de investigación actualización del mundo de las ciencias

\author{
Carlos Gonzalo Aguirre Rodríguez a, Carlos Alfredo Cevallos Monar ${ }^{\text {b; Wimper }}$ \\ Venancio Nivela Zapata ${ }^{c}$
}

Modelo de gestión funcional para el área de salud

Functional management model for the health area

Revista Científica de Investigación actualización del mundo de las Ciencias. Vol. 3

núm., 2, abril, ISSN: 2588-0748, 2018, pp. 614-625

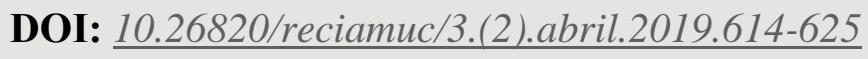

URL: $\underline{\text { http://reciamuc.com/index.php/RECIAMUC/article/view/356 }}$

Código UNESCO: 5909 Administración Publica

Tipo de Investigación: Artículo de Revisión

(C) RECIAMUC; Editorial Saberes del Conocimiento, 2019

Recibido: 15/01/2019

Aceptado: 07/02/2019

Publicado: 01/04/2019

Correspondencia: caguirre@utb.edu.ec

a. Doctor en Ciencias Administrativas; Magister Ejecutivo en Dirección de Empresas con Énfasis en Gerencia Estratégica; Especialista en Gerencia de Proyectos; Diplomado Superior en Gerencia de Marketing; Magister en Docencia y Currículo; Universidad Técnica de Babahoyo; Babahoyo, Ecuador; caguirre@utb.edu.ec

b. Magister en Administración de Empresas; Diploma Superior en Docencia Universitaria; Ingeniero en Electricidad Especialización Electrónica; Universidad Técnica de Babahoyo; Babahoyo, Ecuador; acevallos@utb.edu.ec

c. Doctor en Jurisprudencia y Abogado de los Tribunales y Juzgados de la Republica; Magister en Administración de Empresas; Licenciado en Ciencias Publicas y Sociales; Universidad Técnica de Babahoyo; Babahoyo, Ecuador; wnivelazapata@ hotmail.com 
Carlos Gonzalo Aguirre Rodríguez; Carlos Alfredo Cevallos Monar; Wimper Venancio Nivela

Zapata

\title{
RESUMEN
}

Objetivo: Diseñar estrategias para aplicar el modelo de gestión por procesos en la Maternidad del Gobierno Provincial Autónomo de Los Ríos que mejoren la calidad de sus servicios. Metodología: La muestra fue de 40 encuestados y analizados durante los años 2014-2015 de la población adscrita al Área $N^{\circ}$. 1, Centro Materno Infantil Enrique Ponce Luque - Babahoyo. Fueron consideradas como variables: niveles de conocimiento y estrategias, para el análisis de los datos obtenidos se utilizó estadística descriptiva y plantillas de Excel. Resultados: El modelo de gestión funcional permitió a la maternidad desarrollar sus actividades iniciales en forma adecuada, pero que al momento es necesario generar un cambio en la gestión administrativa y operativa de la entidad, por lo que estiman que la gestión por procesos debidamente socializada y aplicada generaría un mejor nivel de competitividad y estructuración con relación a las unidades de salud del estado. Conclusiones: Se identificaron los factores que afectan el desarrollo y crecimiento organizacional bajo el modelo de gestión funcional que se viene manejando en la Maternidad del Gobierno Autónomo Descentralizado de Los Ríos.

Palabras Claves: Modelo de gestión; Personal médico; Personal paramédico; Gestión por procesos.

\begin{abstract}
Objective: Design strategies to apply the process management model in the Maternity of the Autonomous Provincial Government of Los Ríos that improve the quality of its services. Methodology: The sample was 40 respondents and analyzed during the years 2014-2015 of the population assigned to Area No. 1, Enrique Ponce Luque Maternal and Child Center - Babahoyo. They were considered as variables: levels of knowledge and strategies, for the analysis of the data obtained descriptive statistics and Excel templates were used. Results: The functional management model allowed maternity to develop its initial activities in an adequate manner, but that at the moment it is necessary to generate a change in the administrative and operational management of the entity, so they consider that the management by processes properly socialized and applied would generate a better level of competitiveness and structuring in relation to the
\end{abstract}




\section{Modelo de gestión funcional para el área de salud}

Vol. 3, núm. 2., (2019)

Carlos Gonzalo Aguirre Rodríguez; Carlos Alfredo Cevallos Monar; Wimper Venancio Nivela Zapata

state health units. Conclusions: The factors that affect the development and organizational growth were identified under the functional management model that has been managed in the Maternity of the Decentralized Autonomous Government of Los Ríos.

Key Words: Management model; Medical staff; Paramedical staff; Process management.

\section{Introducción.}

Se debe partir de la conceptualización propia de lo que es un modelo de gestión, el cual se considera como un esquema o marco de referencia para la administración de una organización, por ello, los modelos de gestión pueden ser aplicados no solamente en las empresas y negocios privados, sino también en la administración pública. (Oviedo Rodríguez \& Viejo Placencio, 2014) (Molero \& Panunzio, 2010)

En Ecuador podemos señalar a ciencia cierta que el gobierno en la actualidad, si tiene un modelo de gestión aplicable para las instituciones del sector público en el que se basan para desarrollar sus políticas y acciones, y con el cual pretenden alcanzar sus objetivos.

Existen varias diferenciaciones en la aplicación de estos modelos, sin embargo se puede apreciar que el modelo de gestión que utilizan las organizaciones públicas es totalmente diferente al modelo de gestión del ámbito privado. Mientras este último se basa en la obtención de ganancias económicas, el del sector público pone en juego otras cuestiones, como el bienestar social de la población. (Tejada Zabaleta, 2003) (Pont Vidal, 2016) (Barreda Tamayo, 2007)

En la presente investigación se revisan diferentes situaciones desde la aplicabilidad de un modelo de gestión por procesos que se mantiene en las entidades del sector público, hacia la aplicación del mismo en una institución de salud del sector privado sin fines de lucro, pero que 


\section{Modelo de gestión funcional para el área de salud}

Vol. 3, núm. 2., (2019)

Carlos Gonzalo Aguirre Rodríguez; Carlos Alfredo Cevallos Monar; Wimper Venancio Nivela

Zapata

en sí busca acercarse a lo social antes que a la generación de utilidades, eso es la Maternidad del Gobierno

Provincial Autónomo de Los Ríos, dado su composición como organización regentada por la Fundación "Antonio Basterrechea Irusta" (FABI), que es quien tiene a su cargo la administración de la misma y busca mejorar sus procesos. Como en toda organización también hay otros grupos de interés (empleados, proveedores, administración, etc.) a cuyas necesidades y expectativas también hay que dar respuesta.

Se hace necesario entonces que en base a la gestión de procesos, qué es lo que quieren nuestros clientes y demás grupos de interés, y en función de sus requisitos, identificar, definir y desarrollar los procesos necesarios para conseguir los objetivos establecidos. Por lo que la gestión pública, entendida como la manera en que el Estado obtiene, organiza, maneja y moviliza los recursos escasos con la finalidad de cumplir ciertos objetivos también ha cambiado. En las últimas décadas hemos podido observar que la gestión ha variado desde una gestión burocrática enfocada en los procesos, normas y procedimientos hacia una gestión enfocada en los resultados, de allí la importancia de reorientar la administración de la Maternidad del Gobierno Provincial Autónomo de Los Ríos, hacia nuevos procesos que cumplan con los objetivos de ayuda hacia lo social y no a lo económico.

La gestión por procesos, definida como la metodología corporativa cuyo objetivo es mejorar el desempeño (Eficiencia y Eficacia) de la Organización a través de la entrega de servicios según los procesos del negocio, que se deben diseñar, modelar, organizar, documentar 


\section{Modelo de gestión funcional para el área de salud}

Vol. 3, núm. 2., (2019)

Carlos Gonzalo Aguirre Rodríguez; Carlos Alfredo Cevallos Monar; Wimper Venancio Nivela Zapata

y optimizar de forma continua. Nos referimos al cambio operacional de la organización al migrar de una administración funcional hacia una de administración por procesos.

En el presente trabajo se incursiona en el área de una organización privada, de carácter social, y, que tiene a cargo el cuidado de la salud humana. Por ello se busca definir la aplicación de las diferentes normas, documentos y formatos requeridos para lograr un mayor rendimiento empresarial, bajo el nuevo modelo de gestión que se propone debe asumir esta institución.

\section{Metodología.}

La muestra fue de 40 encuestados y analizados durante los años 2014-2015 de la población adscrita al Área No. 1, Centro Materno Infantil Enrique Ponce Luque - Babahoyo. Fueron consideradas como variables: niveles de conocimiento y estrategias, para el análisis de los datos obtenidos se utilizó estadística descriptiva y plantillas de Excel.

\section{Resultados.}

\section{Pruebas estadísticas aplicadas}

Una vez aplicados los instrumentos para la recolección de los datos de la presente investigación, se procedió a la agrupación de cada repuesta utilizando el programa tecnológico Microsoft Office Excel, estableciendo en primer lugar cuadros que permitieron realizar los gráficos y proyectar el análisis de los mismos, para confrontarlos con la hipótesis pertinente.

Una vez realizado el trabajo de investigación pertinente en las diferentes áreas de servicio de la maternidad, sobre el modelo de gestión funcional que se aplica actualmente, frente al de 


\section{Modelo de gestión funcional para el área de salud}

Vol. 3, núm. 2., (2019)

Carlos Gonzalo Aguirre Rodríguez; Carlos Alfredo Cevallos Monar; Wimper Venancio Nivela

Zapata

gestión por procesos, que mejore la calidad de sus servicios a los pacientes, se procedió al análisis e interpretación de los resultados y se llegó a la conclusión de que la hipótesis: “Diseñadas las estrategias se aplica el modelo de gestión por procesos en la Maternidad del Gobierno Provincial Autónomo de Los Ríos mejorando la calidad de sus servicios", se encuentra verificada de acuerdo a los datos estadísticos obtenidos.

Por lo tanto los procedimientos fueron:

Definida la hipótesis, se hicieron operables los términos o variables, los mismos que nos dieron su aceptación.

- La comprobación de la hipótesis se la realizó mediante análisis estadísticos, el mismo que se fundamentó en la porcentualidad.

- La verificación de la hipótesis también conocida por el diseño de la prueba y consistió en la elección de la técnica de la porcentualidad.

- Mediante la teoría estadística de la porcentualidad se probó el grado de relación y significación de las variables de correlación.

Resumen de encuesta a usuarios internos - Personal médico y paramédico

\begin{tabular}{|c|c|c|c|c|c|c|c|c|}
\hline PREGUNTA & Mucho & $\%$ & Poco & $\%$ & Nada & $\%$ & Total & $\%$ \\
\hline 1.- ¿Conoce usted lo que es el modelo de gestión funcional? & 28 & $70 \%$ & 8 & $20 \%$ & 4 & $10 \%$ & 40 & $100 \%$ \\
\hline $\begin{array}{l}\text { 2.- ¿Participo usted en el desarrollo de la aplicación del modelo de } \\
\text { gestión funcional? }\end{array}$ & 14 & $35 \%$ & 12 & $30 \%$ & 14 & $35 \%$ & 40 & $100 \%$ \\
\hline $\begin{array}{l}\text { 3.- ¿Considera usted que el actual modelo de gestión que mantiene } \\
\text { la } \\
\text { maternidad es obsoleto? }\end{array}$ & 32 & $80 \%$ & 6 & $15 \%$ & 2 & $5 \%$ & 40 & $100 \%$ \\
\hline
\end{tabular}




\section{Modelo de gestión funcional para el área de salud}

Vol. 3, núm. 2., (2019)

Carlos Gonzalo Aguirre Rodríguez; Carlos Alfredo Cevallos Monar; Wimper Venancio Nivela Zapata

\begin{tabular}{|c|c|c|c|c|c|c|c|c|}
\hline $\begin{array}{l}\text { 4.- ¿Usted ha participado en los diseños de las estrategias para } \\
\text { mejorar } \\
\text { el desarrollo organizacional? }\end{array}$ & 14 & $35 \%$ & 12 & $30 \%$ & 14 & $35 \%$ & 40 & $100 \%$ \\
\hline $\begin{array}{l}\text { 5.- ¿Considera usted que la maternidad goza de buena } \\
\text { competitividad } \\
\text { frente a las unidades de salud locales? }\end{array}$ & 16 & $40 \%$ & 10 & $25 \%$ & 14 & $35 \%$ & 40 & $100 \%$ \\
\hline $\begin{array}{l}\text { 6.- ¿Creé que es necesario desarrollar nuevas estrategias } \\
\text { institucionales } \\
\text { que mejoren la competitividad de la organización? }\end{array}$ & 32 & $80 \%$ & 6 & $15 \%$ & 2 & $5 \%$ & 40 & $100 \%$ \\
\hline $\begin{array}{l}\text { 7.- ¿Estima usted necesario que la maternidad cuente con un nuevo } \\
\text { modelo de gestión administrativo? }\end{array}$ & 34 & $85 \%$ & 4 & $10 \%$ & 2 & $5 \%$ & 40 & $100 \%$ \\
\hline 8.- ¿Estaría dispuesto a capacitarse en un nuevo modelo de gestión? & 34 & $85 \%$ & 2 & $5 \%$ & 4 & $10 \%$ & 40 & $100 \%$ \\
\hline 9.- ¿Conoce usted lo que es el modelo de gestión por proceso? & 20 & $50 \%$ & 10 & $25 \%$ & 10 & $25 \%$ & 40 & $100 \%$ \\
\hline $\begin{array}{l}\text { 10.- ¿Participaría activamente en la aplicación del modelo de } \\
\text { gestión } \\
\text { por procesos en la maternidad? }\end{array}$ & 30 & $75 \%$ & 10 & $15 \%$ & 4 & $10 \%$ & 44 & $100 \%$ \\
\hline $\begin{array}{l}\text { 11.- ¿Estima usted que el área en la que labora se beneficiaría de la } \\
\text { aplicación del modelo de gestión por procesos? }\end{array}$ & 32 & $80 \%$ & 6 & $15 \%$ & 2 & $5 \%$ & 40 & $100 \%$ \\
\hline $\begin{array}{l}\text { 12.- ¿Creé usted que la maternidad mejoraría su nivel } \\
\text { competitividad con la aplicación del modelo de gestión por } \\
\text { procesos? }\end{array}$ & 30 & $75 \%$ & 6 & $15 \%$ & 4 & $10 \%$ & 40 & $100 \%$ \\
\hline
\end{tabular}

Resumen de Encuesta a usuarios internos - Personal administrativo y de servicio

\begin{tabular}{|c|c|c|c|c|c|c|c|c|}
\hline PREGUNTA & Mucho & $\%$ & Poco & $\%$ & Nada & $\%$ & Total & $\%$ \\
\hline 1.- ¿Conoce usted lo que es el modelo de gestión funcional? & 10 & $63 \%$ & 4 & $25 \%$ & 2 & $13 \%$ & 16 & $100 \%$ \\
\hline $\begin{array}{l}\text { 2.- ¿Participo usted en el desarrollo de la aplicación del modelo de } \\
\text { gestión funcional? }\end{array}$ & 8 & $50 \%$ & 6 & $38 \%$ & 2 & $13 \%$ & 16 & $100 \%$ \\
\hline $\begin{array}{l}\text { 3.- ¿Considera usted que el actual modelo de gestión que mantiene la } \\
\text { maternidad es obsoleto? }\end{array}$ & 12 & $75 \%$ & 2 & $13 \%$ & 2 & $13 \%$ & 16 & $100 \%$ \\
\hline $\begin{array}{l}\text { 4.- ¿Usted ha participado en los diseños de las estrategias para mejorar el } \\
\text { desarrollo organizacional? }\end{array}$ & 9 & $56 \%$ & 6 & $38 \%$ & 1 & $6 \%$ & 16 & $100 \%$ \\
\hline $\begin{array}{l}\text { 5.- ¿Considera usted que la maternidad goza de buena competitividad } \\
\text { frente a las unidades de salud locales? }\end{array}$ & 7 & $44 \%$ & 6 & $38 \%$ & 3 & $19 \%$ & 16 & $100 \%$ \\
\hline
\end{tabular}




\begin{tabular}{|c|c|c|c|c|c|c|c|c|}
\hline $\begin{array}{l}\text { 6.- ¿Creé que es necesario desarrollar nuevas estrategias institucionales } \\
\text { que mejoren la competitividad de la organización? }\end{array}$ & 12 & $75 \%$ & 2 & $13 \%$ & 2 & $13 \%$ & 16 & $100 \%$ \\
\hline $\begin{array}{l}\text { 7.- ¿Estima usted necesario que la maternidad cuente con un nuevo } \\
\text { modelo de gestión administrativo? }\end{array}$ & 14 & $88 \%$ & 1 & $6 \%$ & 1 & $6 \%$ & 16 & $100 \%$ \\
\hline 8.- ¿Estaría dispuesto a capacitarse en un nuevo modelo de gestión? & 14 & $88 \%$ & 1 & $6 \%$ & 1 & $6 \%$ & 16 & $100 \%$ \\
\hline 9.- ¿Conoce usted lo que es el modelo de gestión $p$ & 8 & $50 \%$ & 6 & $38 \%$ & 2 & $13 \%$ & 16 & $100 \%$ \\
\hline $\begin{array}{l}\text { 10.- ¿Participaría activamente en la aplicación del modelo de gestión por } \\
\text { procesos en la maternidad? }\end{array}$ & 13 & $81 \%$ & 6 & $13 \%$ & 1 & $6 \%$ & 20 & $100 \%$ \\
\hline $\begin{array}{l}\text { 11.- ¿Estima usted que el área en la que labora se beneficiaría de la } \\
\text { aplicación del modelo de gestión por procesos? }\end{array}$ & 12 & $75 \%$ & 3 & $19 \%$ & 1 & $6 \%$ & 16 & $100 \%$ \\
\hline $\begin{array}{l}\text { 12.- ¿Creé usted que la maternidad mejoraría su nivel de competitividad } \\
\text { con la aplicación del modelo de gestión por procesos? }\end{array}$ & 12 & $75 \%$ & 2 & $13 \%$ & 2 & $13 \%$ & 16 & $100 \%$ \\
\hline
\end{tabular}

\section{Resumen de Encuesta a usuarios externos}

\begin{tabular}{|c|c|c|c|c|c|c|c|c|}
\hline PREGUNTA & Mucho & $\%$ & Poco & $\%$ & Nada & $\%$ & Total & $\%$ \\
\hline $\begin{array}{l}\text { 1.- ¿Considera usted que el actual modelo de gestión que } \\
\text { mantiene la maternidad es obsoleto? }\end{array}$ & 32 & $59 \%$ & 16 & $30 \%$ & 6 & $11 \%$ & 54 & $100 \%$ \\
\hline $\begin{array}{l}\text { 2.- ¿Considera usted que la maternidad goza de buena } \\
\text { competitividad frente a las unidades de salud locales? }\end{array}$ & 30 & $56 \%$ & 14 & $26 \%$ & 10 & $19 \%$ & 54 & $100 \%$ \\
\hline $\begin{array}{l}\text { 3.- ¿Creé que es necesario desarrollar nuevas estrategias } \\
\text { institucionales que mejoren la competitividad de la } \\
\text { organización? }\end{array}$ & 38 & $70 \%$ & 10 & $19 \%$ & 6 & $11 \%$ & 54 & $100 \%$ \\
\hline $\begin{array}{l}\text { 4.- ¿Estima usted necesario que la maternidad cuente con un } \\
\text { nuevo modelo de gestión administrativo? }\end{array}$ & 46 & $85 \%$ & 5 & $9 \%$ & 3 & $6 \%$ & 54 & $100 \%$ \\
\hline 5.- ¿Conoce usted lo que es el modelo de gestión por proceso? & 26 & $48 \%$ & 15 & $28 \%$ & 13 & $24 \%$ & 54 & $100 \%$ \\
\hline $\begin{array}{l}\text { 6.- ¿Participaría activamente en la aplicación del modelo de } \\
\text { gestión por procesos en la maternidad? }\end{array}$ & 45 & $83 \%$ & 8 & $15 \%$ & 1 & $2 \%$ & 54 & $100 \%$ \\
\hline \multirow{2}{*}{$\begin{array}{l}\text { 7.- ¿Creé usted que la maternidad mejoraría su nivel de } \\
\text { competitividad con la aplicación del modelo de gestión por } \\
\text { procesos? }\end{array}$} & 46 & $85 \%$ & 6 & $11 \%$ & 2 & $4 \%$ & 54 & $100 \%$ \\
\hline & Muy & $\%$ & $\begin{array}{c}\text { Poco } \\
\text { importa } \\
\text { nte }\end{array}$ & $\%$ & $\begin{array}{l}\text { Nada } \\
\text { impor } \\
\text { tante }\end{array}$ & $\%$ & Total & $\%$ \\
\hline
\end{tabular}




\section{Modelo de gestión funcional para el área de salud}

Vol. 3, núm. 2., (2019)

Carlos Gonzalo Aguirre Rodríguez; Carlos Alfredo Cevallos Monar; Wimper Venancio Nivela Zapata

\begin{tabular}{ll|l|l|l|l|l|l|l|l|}
$\begin{array}{l}\text { 8.- ¿En su calidad de usuario en la gestión de la maternidad } \\
\text { como se considera? }\end{array}$ & 29 & $54 \%$ & 14 & $26 \%$ & 11 & $20 \%$ & 54 & $100 \%$ \\
\hline
\end{tabular}

\section{Entrevista a directivos}

\begin{tabular}{|c|c|c|c|c|}
\hline Pregunta: & Mucho & Poco & Nada & Comentarios: \\
\hline $\begin{array}{l}\text { 1.- ¿Participo usted en el desarrollo de la aplicación del modelo de } \\
\text { gestión funcional? }\end{array}$ & $\mathbf{X}$ & & & $\begin{array}{c}\text { HACE MUCHOS AÑOS ATRÁS SE } \\
\text { PARTICIPO EN ELLO }\end{array}$ \\
\hline $\begin{array}{l}\text { 2.- ¿Considera usted que el actual modelo de gestión que mantiene la } \\
\text { maternidad es obsoleto? }\end{array}$ & & $\mathbf{X}$ & & ES NECESARIO UN CAMBIO \\
\hline $\begin{array}{l}\text { 3.- ¿Usted ha participado en los diseños de las estrategias para mejorar } \\
\text { el desarrollo organizacional? }\end{array}$ & & $\mathbf{X}$ & & $\begin{array}{l}\text { DEBEN DEFINIRSE NUEVAS } \\
\text { ESTRATEGIAS }\end{array}$ \\
\hline $\begin{array}{l}\text { 4.- ¿Considera usted que la maternidad goza de buena competitividad } \\
\text { frente a las unidades de salud locales? }\end{array}$ & & $\mathbf{X}$ & & $\begin{array}{l}\text { LAS UNIDADES DE SALUD } \\
\text { PÚBLICAS NOS ABSORVEN }\end{array}$ \\
\hline $\begin{array}{l}\text { 5.- ¿Creé que es necesario desarrollar nuevas estrategias } \\
\text { institucionales } \\
\text { que mejoren la competitividad de la organización? }\end{array}$ & $\mathbf{X}$ & & & $\begin{array}{c}\text { ES NECESARIO GENERAR } \\
\text { VALOR } \\
\text { AGREGADO A NUESTROS } \\
\text { SERVICIOS }\end{array}$ \\
\hline $\begin{array}{l}\text { 6.- ¿Estima usted necesario que la maternidad cuente con un nuevo } \\
\text { modelo de gestión administrativo? }\end{array}$ & $\mathbf{X}$ & & & $\begin{array}{l}\text { POR LA NECESECIDAD DE } \\
\text { MODERNIZAR LA ENTIDAD }\end{array}$ \\
\hline 7.- ¿Conoce usted lo que es el modelo de gestión por proceso? & & $\mathbf{X}$ & & POCO SE CONOCE AL RESPECTO \\
\hline $\begin{array}{l}\text { 8.- ¿Participaría activamente en la aplicación del modelo de gestión } \\
\text { por } \\
\text { procesos en la maternidad? }\end{array}$ & $\mathbf{X}$ & & & $\begin{array}{l}\text { A FIN DE MODERNIZAR LOS } \\
\text { SERVICIOS SI PARTICIPARÍA }\end{array}$ \\
\hline $\begin{array}{l}\text { 9.- ¿Creé usted que la maternidad mejoraría su nivel de competitividad } \\
\text { con la aplicación del modelo de gestión por procesos? }\end{array}$ & $\mathbf{X}$ & & & $\begin{array}{l}\text { UN NUEVO MODELO TRAE } \\
\text { CONSIGO EL CAMBIO } \\
\text { INSTITUCIONAL }\end{array}$ \\
\hline
\end{tabular}




\section{Modelo de gestión funcional para el área de salud}

Vol. 3, núm. 2., (2019)

Carlos Gonzalo Aguirre Rodríguez; Carlos Alfredo Cevallos Monar; Wimper Venancio Nivela

Zapata

Análisis: Los sujetos de observación, en este caso a los directivos que se aplicó el instrumento entrevista, establecen que el modelo de gestión funcional permitió a la maternidad desarrollar sus actividades inciales en forma adecuada, pero que al momento es necesario generar un cambio en la gestión administrativa y operativa de la entidad, por lo que estiman que la gestión por procesos debidamente socializada y aplicada generaría un mejor nivel de competitividad y estructuración con relación a las unidades de salud del estado.

Interpretación: Los directivos de la maternidad consideran que es necesario aplicar un nuevo modelo de gestión, en este caso el que se aplica por las otras organizaciones de salud del sector público a fin de generar una mayor dinamia en la atención a los usuarios de la maternidad.

\section{Conclusiones.}

Se identificaron los factores que afectan el desarrollo y crecimiento organizacional bajo el modelo de gestión funcional que se viene manejando en la Maternidad del Gobierno Autónomo Descentralizado de Los Ríos.

Con la aplicación de los instrumentos aplicados tanto a directivos como al resto del personal y usuarios se pudo demostrar porque la Maternidad del Gobierno Autónomo Descentralizado de Los Ríos, siendo un ente de carácter social no gubernamental, debe asumir como suyo el modelo de gestión por procesos para mejorar su dinámica de competitividad frente a las unidades de salud de su entorno.

Se determinan varias estrategias para aplicar el modelo de gestión por procesos en la Maternidad del Gobierno Autónomo Descentralizado de Los Ríos, lo que permitirá mejorar el 


\section{Modelo de gestión funcional para el área de salud}

Vol. 3, núm. 2., (2019)

Carlos Gonzalo Aguirre Rodríguez; Carlos Alfredo Cevallos Monar; Wimper Venancio Nivela Zapata

sistema organizacional de este tipo de entidad dedicada a la salud y con ello brinde una atención de calidad a sus usuarios.

\section{Recomendaciones.}

La identificación de los factores que afectan el desarrollo y crecimiento organizacional bajo el modelo de gestión funcional, permitieron definir varios aspectos que deben asumir los directivos para aplicar un nuevo modelo de gestión denominado como de proceso.

Los instrumentos aplicados tales como encuesta y entrevistas permitieron demostrar porque la Maternidad del Gobierno Autónomo Descentralizado de Los Ríos, siendo un ente de carácter social no gubernamental debe asumir el modelo de gestión por procesos.

Deben aplicarse las estrategias definidas y presentadas en la alternativa de solución que permita asumir el modelo de gestión por procesos en la Maternidad del Gobierno Autónomo Descentralizado de Los Ríos.

\section{Bibliografía.}

Barreda Tamayo, H. (2007). Características distintivas en la gestion del servicio educativo. Revista Gestão Universitária na América Latina - GUA, 1(1), 2-15.

Molero, T., \& Panunzio, A. (2010). Gestión de la calidad de atención en laboratorios clínicos de hospitales públicos en Maracaibo, Venezuela. Revista de Salud Pública, 12(4), 658-668.

Oviedo Rodríguez, M., \& Viejo Placencio, L. (2014). Estudio comparativo del modelo de atención ideal y lo real de los servicios de salud que proporcionen mejoramiento de calidad a los pacientes del subcentro de salud de Pijullo. Babahoyo: UTB.

Pont Vidal, J. (2016). Modelos innovadores de administración y gestión pública: Hacia la emergencia de nuevos paradigmas. Gestión y Análisis de Políticas Públicas, 16(1), 4-22. 
Modelo de gestión funcional para el área de salud

Vol. 3, núm. 2., (2019)

Carlos Gonzalo Aguirre Rodríguez; Carlos Alfredo Cevallos Monar; Wimper Venancio Nivela

Zapata

Tejada Zabaleta, A. (2003). Los modelos actuales de gestión en las organizaciones. gestión del talento, gestion del conocimiento y gestión por competencias. Psicología desde el Caribe, 12(Jul), 115-133.

$$
\text { (C) }(\mathcal{Q} \Theta()
$$

RECONOCIMIENTO-NOCOMERCIAL-COMPARTIRIGUAL

CC BY-NC-SA

ESTA LICENCIA PERMITE A OTROS ENTREMEZCLAR, AJUSTAR Y CONSTRUIR A PARTIR DE SU OBRA CON FINES NO

COMERCIALES, SIEMPRE Y CUANDO LE RECONOZCAN LA AUTORÍA Y SUS NUEVAS CREACIONES ESTÉN BAJO UNA LICENCIA CON LOS MISMOS TÉRMINOS. 